\title{
Highly catalytically active CeO2-x based heterojunction nanostructures with mixed micro/meso-porous architectures
}

Sajjad S. Mofarah,* Luisa Schreck, Claudio Cazorla, Xiaoran Zheng, Esmaeil Adabifiroozjaei, Constantine Tsounis, Jason Scott, Reza Shahmiri, Yin Yao, Roozbeh Abbasi, Kourosh Kalantar-Zadeh, Yuan Wang, Hamidreza Arandiyan, Leigh Sheppard, Esmail Doustkhah, Pramod Koshy, and Charles C. Sorrell*

ABSTRACT: Achieving high densities of accessible active sites in catalysts, which depend principally on the architecture of nanostructures, is critical to obtain enhanced performance. The present work introduces a template-free, high-yield, and flexible approach to fabricate 3D, mesoporous, $\mathrm{CeO}_{2-x}$ nanostructures in centimeter-scale that are comprised of extremely thin holey 2D nanosheets. The method involves conversion of a stacked, 2D, Ce-based coordination polymer by controlling the removal kinetics of organic species. The resultant polycrystalline $2 \mathrm{D}-3 \mathrm{D} \mathrm{CeO}_{2-x}$ exhibits a large density of defects as well as outstanding surface areas of $251 \mathrm{~m}^{2} \mathrm{~g}^{-1}$. This mesoporous nanomaterial yields superior $\mathrm{CO}$ conversion performance $\left(\mathrm{T}_{90 \%}=148^{\circ} \mathrm{C}\right)$. Further improvements in catalysis were attained by synthesis $\mathrm{CeO}_{2-x}$-based transition metal oxides (TMOs) hetero-nanostructures, for which structural analyses and first principles simulations revealed active sites associated with the TMOs. This versatile fabrication technique delivers new pathways to engineer nanostructures and advance their functionalities for catalysis.

KEYWORDS: Holey Two-Dimensional, Defect-rich $\mathrm{CeO}_{2}$, Porous Heterojunction Nanostructure, Ce-based Coordination Polymers

\section{INTRODUCTION}

Three dimensional (3D) mesoporous metal oxides (MOs) hold great promise in catalysis through increasing accessibility of the active sites relative to bulk materials. Nonetheless, the use of sacrificial templates, which generally is employed to introduce the pores and allow the formation of the 3D structures, makes the fabrication process complex and of low efficiency. Certain shortcomings also apply to various etching techniques for creating pores in 3D scaffolds $^{1,2}$. In porous structures, the walls of the 3D scaffolds limit the number of active sites to only the surface and subsurface of these 3D units. The recently developed techniques involving the creation of nanoholes in two dimensional (2D) MOs has facilitated the accessibility of active sites by exposing greater surface area, ${ }^{3-5}$ thus making these materials attractive for surface-sensitive applications in energy, sensing, and heterogeneous catalysis. ${ }^{6-8}$ Further, the intrinsic polycrystallinity of these holey 2D structures can overcome the critical shortcoming of irreversible restacking of pristine 2D nanosheets. ${ }^{9}$, 10 Recent advances in the synthesis of polycrystalline holey 2D MOs include the heterogeneous deposition of mixed transition metal oxides (TMOs) on graphene nanosheets as a sacrificial template ${ }^{11}$ and the synthesis of holey TMOs by etching pristine 2D TMOs. ${ }^{12-14}$ Despite considerable improvements in the performances of these nanostructures, challenges, such as complex synthesis procedures, use of 
surfactants and templates, excessive nanosheet thicknesses, ${ }^{15}$ and low yields, limit their practical applicabilities. Unlike TMOs, there are few reports of pristine 2D rare earth oxides (REOs), none of which exhibit intrinsically layered structures. Consequently, complex methods of self-assembly have been applied as an alternative to exfoliation, which typically is applied to convert layered materials to 2D nanostructures. ${ }^{16,}{ }^{17}$ Although such nanostructures have shown outstanding catalytic and photocatalytic performance, ${ }^{18}$ they have the disadvantage of low-yield production. ${ }^{19}$

The present work reports a new synthesis route to fabricate multidimensional 2D-3D structures consisting of 2D holey nanosheets of $\mathrm{CeO}_{2-x}$ (or TMO-decorated $\mathrm{CeO}_{2-\mathrm{x}}$ ) that stack to form mesoporous 3D nanostructures with enhanced catalytic functionalities.

\section{- RESULTS}

Fabrication of Porous $\mathrm{CeO}_{2}$ Hexagonal tubes Derived from CeriumBased Coordination Polymer (Ce-CP)

In order to synthesize the 2D-3D $\mathrm{CeO}_{2-x}$, a novel Ce-CP, which is the sole precursor, was fabricated electrochemically at room temperature. ${ }^{9}$ This resulted in the formation of free-standing hexagonal tubes grown vertically on fluorinedoped tin oxide (FTO) substrates. The Ce-CP has the molecular formula of $\mathrm{Ce}(\mathrm{TCA})_{2}(\mathrm{OH})_{2} \cdot 2 \mathrm{H}_{2} \mathrm{O}$ (TCA - trichloroacetate) and the $\beta$-axis view of the structure is shown schematically in Figure $1 \mathrm{a}$, where the unit cell with triclinic structure (space group $\mathrm{P} \overline{1}$ ) is also defined. The Ce ions are covalently bonded to the $\mathrm{O}^{2-}$ ions of $\mathrm{H}_{2} \mathrm{O}, \mathrm{OH}^{-}$, and TCA, thereby forming stacked layers of thickness $1.04 \mathrm{~nm}$ ( $\alpha$-spacing). However, the terminating $\mathrm{Cl}^{-}$ions of TCA at the opposite interfaces are electrostatically attracted to protons, as evidenced from zeta potential $(\xi)$ measurements (Figure S1). It is significant that the Ce-CP can be transformed into $\mathrm{CeO}_{2}$, shown in Figure $1 \mathrm{~b}$, through simple oxidation. This transformation is shown schematically in Figure 1c, where a dense distribution of nanopores results from TCA removal and formation of a porous $\mathrm{CeO}_{2}$ nanotube (Ce-NT); the obtained Ce-NT is shown by scanning electron microscopy (SEM) imaging (Figure ld-f). 

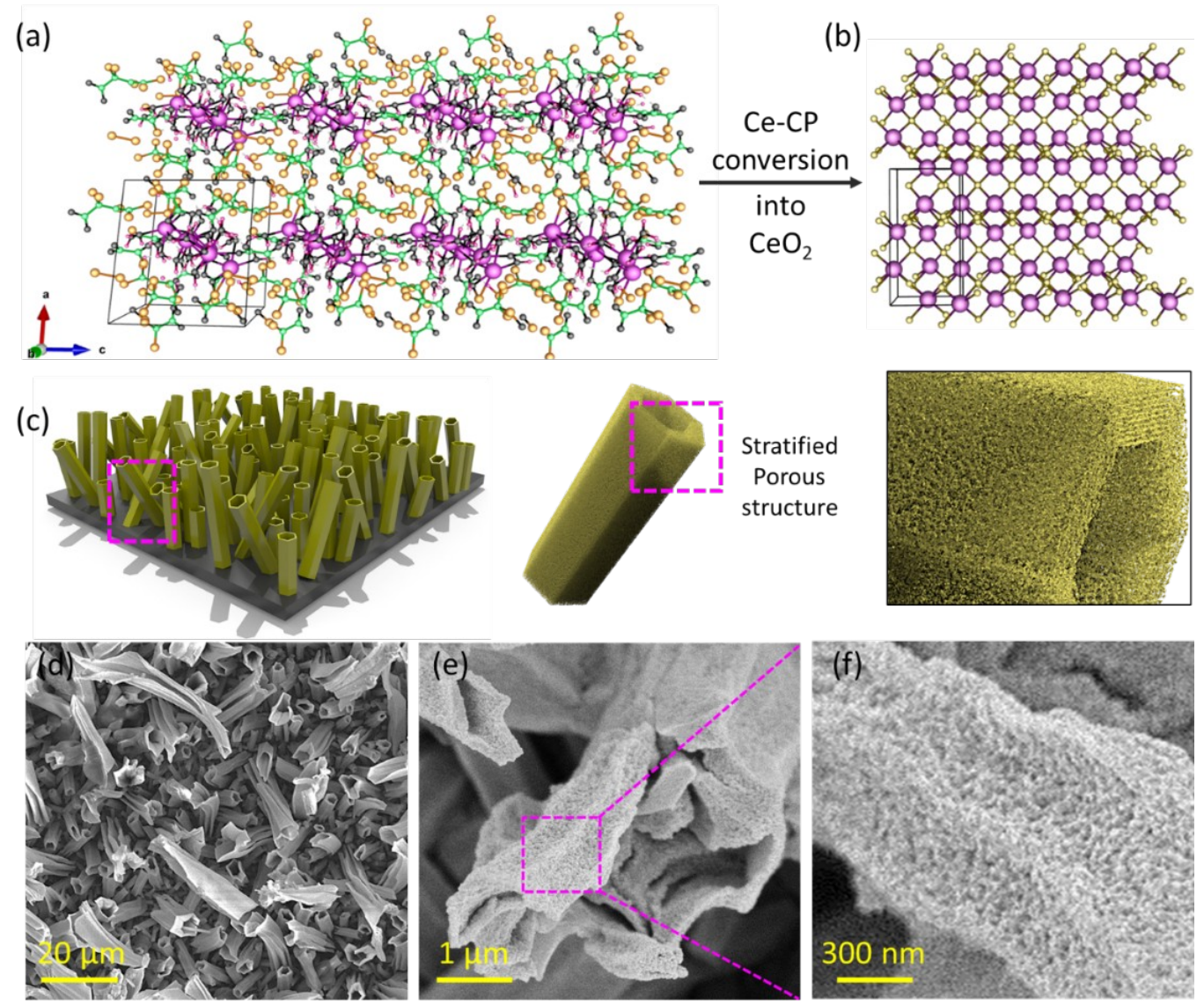

Figure 1. (a) Schematic of $\beta$-axis projection of Ce-CP molecule; (b) schematic of $\mathrm{CeO}_{2}$ molecule; (c) schematic and (d-f) SEM images of porous $\mathrm{CeO}_{2}$ nanotubes on FTO substrates.

Fabrication of 2D-3D $\mathrm{CeO}_{2-x}$ Scaffold Through Exfoliation of Ce-CP. The simple exfoliation and rapid oxidation of the Ce-CP provide the means of tailoring the resultant $\mathrm{CeO}_{2}$ architectures. The formation of 2D-3D oxides involves a twostep process of exfoliation of the bulk-layered Ce-CP in polar triethanolamine (TEA), followed by heat treatment the suspension at $\sim 450^{\circ} \mathrm{C}$ (heating rate $3^{\circ} \mathrm{C} / \mathrm{min}$ ) in air, as shown in Figure $2 \mathrm{a}$. The exfoliation results in a stable colloidal system $(\xi=+34 \mathrm{~V})$ of individual Ce-CP monolayers (Figure S1). The heating process leads to conversion of the $\mathrm{Ce}-\mathrm{CP}$ layers into $\mathrm{CeO}_{2-x}$ through evaporation of the interlayer-trapped TEA, resulting in a mesoporous 3D structure. The importance of the kinetics in the engineering of the final nanostructures (Figure S2) is shown by the effects of the heating rate. Figure $2 \mathrm{~b}, \mathrm{c}$ shows that a slow heating rate $\left(0.2^{\circ} \mathrm{C} / \mathrm{min}\right)$ allowed the laminar evaporation of TEA, resulting in a stacked solid macrolayer of $\mathrm{CeO}_{2-\mathrm{x}}$ covered by intact hemispheres (Figure S2a). An intermediate heating rate $\left(1.0^{\circ} \mathrm{C} / \mathrm{min}\right)$ caused fracture of the hemispheres owing to gas exudation; however, the hemispherical shapes were retained (Figure S2b, c). In contrast, fast heating rates $\left(3.0^{\circ} \mathrm{C} / \mathrm{min}\right.$ ) caused energetic volumetric evaporation of TEA, nanostructural disruption, and formation of thick mesoporous macrolayers (Figure $2 \mathrm{~d}$, e). The significance of TEA in exfoliation is highlighted by contrasting with the conventional oxidation of metal-based coordination polymers (MCPs), where the original microstructure is retained ${ }^{20-22}$. Higher magnifications (Figure 2f-h) reveal nanostructures consisting of clustered 2D nanosheets in 3D scaffold morphology. High-angle annular dark field (HAADF) 
imaging and the selected area diffraction (SAED) pattern (Figure 2i) illustrates the polycrystallinity nature of the nanosheets, consisting of $\mathrm{CeO}_{2}$ crystallite sizes of 2-4 $\mathrm{nm}$ and high concentrations of pores sizes of 3-5 $\mathrm{nm}$. Energy dispersive Xray (EDX) elemental mapping of the holey nanosheet (Figure $2 \mathrm{j}$ ) illustrates homogenous distributions of cerium and oxygen. Atomic force microscopy (AFM) imaging (Figure $2 \mathrm{k}$ ) shows an example of a nanosheet with a thickness of $\sim 4 \mathrm{~nm}$, which represents $\sim 8$ unit cells of cubic $\mathrm{CeO}_{2}$, although the average thickness of the sheets was $\sim 15 \mathrm{~nm}$, which still represents remarkably thin holey nanosheets.

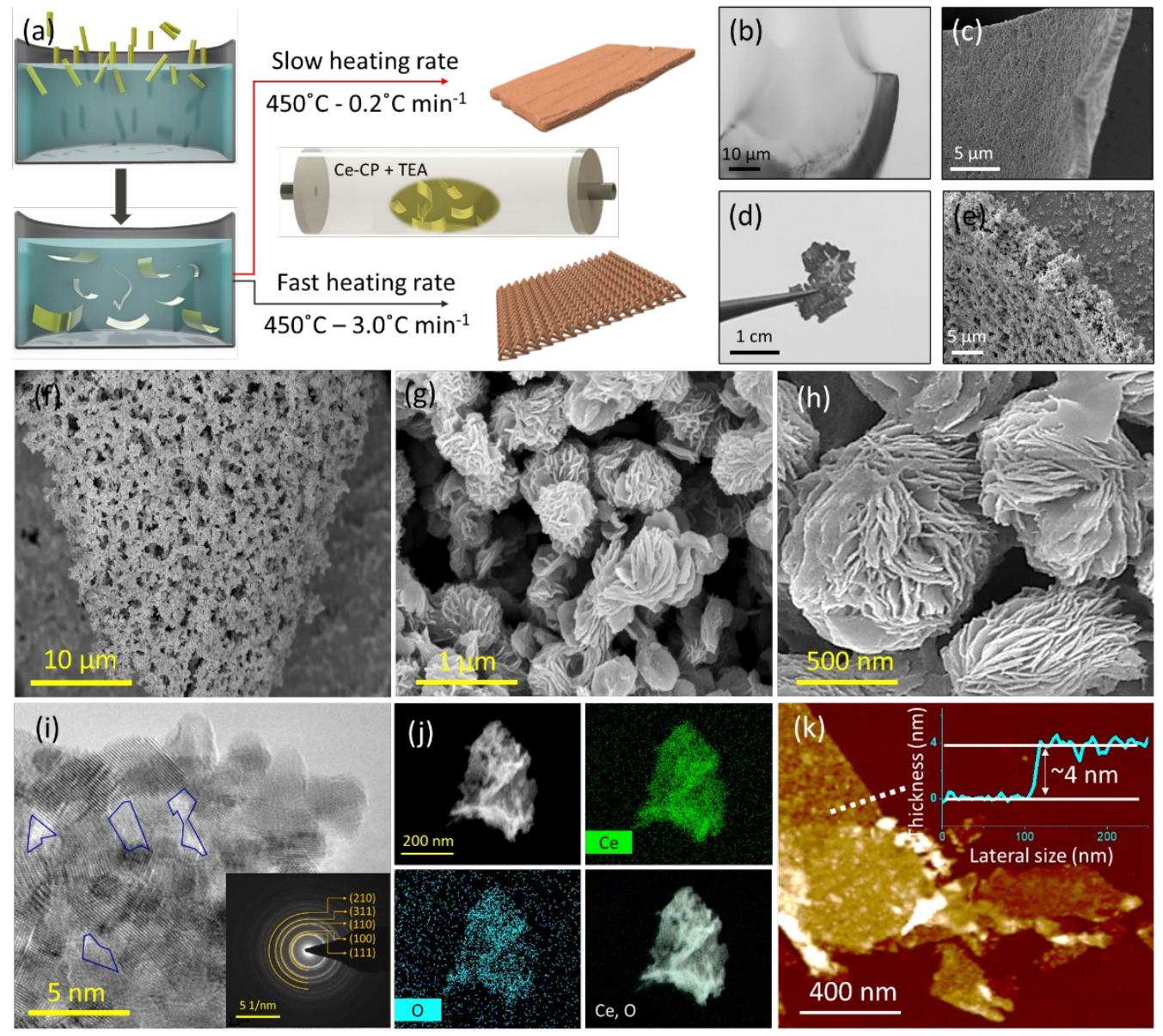

Figure 2. (a) Schematic of two-step process of Ce-CP nanotube exfoliation in stirred TEA solution and oxidation at $450^{\circ} \mathrm{C}$ in air into stacked $\mathrm{CeO}_{2-x}$ macrolayers. (b-e) $\mathrm{CeO}_{2-x}$ morphologies derived from Ce-CP. ( $f-h$ ) SEM images of $2 \mathrm{D}-3 \mathrm{D} \mathrm{CeO}_{2-x}$. (i) HAADF image and SAED of holey $2 \mathrm{D} \mathrm{CeO}_{2-x}$ nanosheet (holes outlined). (j) EDX elemental mapping of holey 2D $\mathrm{CeO}_{2-x}$ nanosheet. (K) AFM image (step height in white dotted line) and corresponding height profile of holey $2 \mathrm{D} \mathrm{CeO}_{2-x}$ nanosheet.

The wide-ranging applicability of the fabrication process is demonstrated by the one-step synthesis of various heterogeneous nanostructures through the addition of water-soluble transition metal salts $\left(\mathrm{CuNO}_{3}\right.$ or $\left.\mathrm{Mn}\left(\mathrm{NO}_{3}\right)_{2}\right)$ during the $\mathrm{Ce}-\mathrm{CP}$ exfoliation step. Subsequent transformation resulted in the formation of 2D-3D $\mathrm{CeO}_{2-x}$ decorated homogeneously with $\mathrm{CuO}(\mathrm{Ce}-\mathrm{Cu})$ or $\mathrm{MnO}(\mathrm{Ce}-\mathrm{Mn})$ of unchanged morphologies, in common with the pristine $2 \mathrm{D}-3 \mathrm{D} \mathrm{CeO}_{2-x}$. Characterization data for the nanostructural features of the $\mathrm{Ce}-\mathrm{Cu}$ and $\mathrm{Ce}-\mathrm{Mn}$ are given in Figures S4-S7. 
Defect Analysis of 2D-3D $\mathrm{CeO}_{2-x}$ and Heterojunction Nanostructures. Figure 3 illustrates the results for structural, defect, and surface analyses of the nanostructures. As shown in Figure $3 \mathrm{a}$, the $\mathrm{Ce}^{3+}$ concentration $\left(\left[\mathrm{Ce}^{3+}\right]\right)$ of the mesoporous 2D-3D $\mathrm{CeO}_{2-x}$ was determined by X-ray photoelectron spectroscopy (XPS) to be 27 at\% $\left(\left[V_{O}^{* *}\right]=13.5\right.$ at\%) using the standard correlation for $\left[\mathrm{Ce}^{3+}\right] .^{23}$ This level can be compared with the data for the electron energy loss spectroscopy (EELS) data shown in Figure 3b. Applying the linear relationship between the $M_{5} / M_{4}$ peak intensity ratio and the $\left[V_{O}^{*}\right],{ }^{24,25}$ a value of 12.8 at\% was obtained for the latter, which is in relatively good agreement with that calculated from $\left[\mathrm{Ce}^{3+}\right]$. The laser Raman microspectroscopy spectra in Figure $3 c$ reveal the $\mathrm{F}_{2} \mathrm{~g}$ vibrational mode of $\mathrm{Ce}-\mathrm{O}$ at $\sim 464 \mathrm{~cm}^{-1}$, confirming the presence of $\mathrm{CeO}_{2} \cdot{ }^{26}$ The spectra for $\mathrm{Cu}-\mathrm{Ce}$ and $\mathrm{Mn}$-Ce reveal asymmetrical peak broadening of the $\mathrm{F}_{2} \mathrm{~g}$ peak at $\sim 464 \mathrm{~cm}^{-1}$ owing to quantum confinement effects. ${ }^{26,}{ }^{27}$ There also are two additional peaks at $\sim 580 \mathrm{~cm}^{-1}$ and $\sim 1147 \mathrm{~cm}^{-1}$ for all three materials, which are attributed to the defect-induced mode (D) and $2^{\text {nd }}$ order longitudinal optical mode (2LO), respectively, associated with $V_{O_{0}}{ }^{26,28,29}$ The XPS data for the TMOdecorated $\mathrm{CeO}_{2-x}$ in Figure 3d,e reveal shoulder peaks (green), which are indicative of $\mathrm{Mn}^{2+}$ and $\mathrm{Cu}^{2+}$ satellites, supporting the presence of $\mathrm{MnO}$ (Figures S4 and S5) and CuO (Figures S6 and S7), ${ }^{30,31}$ as confirmed by the associated SEM and EDX data. The Brunauer-Emmett-Teller (BET) specific surface areas (SSA) were determined to be $51,251,230$, and $160 \mathrm{~m}^{2} \cdot \mathrm{g}^{-1}$ for the $\mathrm{Ce}-\mathrm{NT}, \mathrm{CeO}_{2-x}, \mathrm{Mn}-\mathrm{Ce}$, and $\mathrm{Cu}-\mathrm{Ce}$ nanostructures, respectively, as shown in Figure $3 \mathrm{f}$. The sizes and volumes of the pores are also tabulated (Table S1). The significant effect of exfoliation on the increase in the SSA and the pore volume is shown by the respective fourfold and eightfold increases for the Ce-NT and the 2D-3D $\mathrm{CeO}_{2-x}$. The higher SSA for $\mathrm{Mn}$-Ce relative to that of $\mathrm{Cu}-\mathrm{Ce}$ is attributed to the formation of a compact laminated nanostructure (Figure S6) for the latter compared to the clustered 2D nanosheets in 3D spherical morphology for the former (Figure S4). 

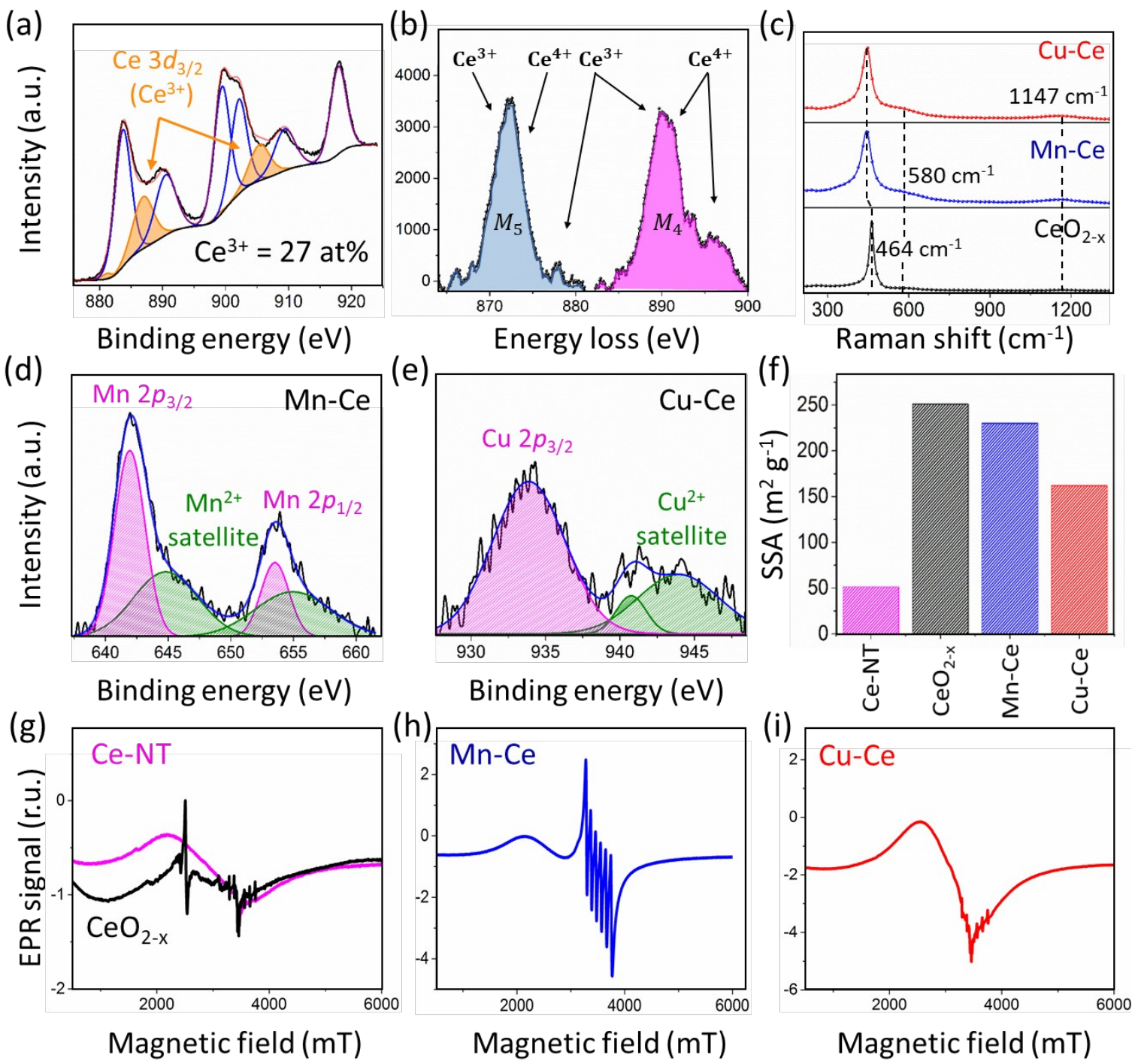

Figure 3. Characterization of nanostructures: (a) XPS Ce 3d spectrum of 2D$3 \mathrm{D} \mathrm{CeO}_{2-x}$ (b) EELS spectrum of 2D-3D $\mathrm{CeO}_{2-x}$. (c) Laser Raman microspectra 2D$3 \mathrm{D} \mathrm{CeO}_{2-x}, \mathrm{Mn}-\mathrm{Ce}$, Cu-Ce. (d) XPS Mn $2 \mathrm{p}$ spectrum of Mn-Ce. (d) XPS $\mathrm{Cu} 2 \mathrm{p}$ spectrum of $\mathrm{Cu}-\mathrm{Ce}$. (f) SSAs of Ce-NT, 2D-3D $\mathrm{CeO}_{2-x}, \mathrm{Mn}-\mathrm{Ce}, \mathrm{Cu}-\mathrm{Ce} .(\mathrm{g}-\mathrm{i}) \mathrm{EPR}$ spectra of Ce-NT, 2D-3D CeO $2-x, \mathrm{Mn}-\mathrm{Ce}, \mathrm{Cu}-\mathrm{Ce}$.

Structural defects were probed by electron paramagnetic resonance (EPR) analyses, as shown in Figure $3 \mathrm{~g}$-i. For the Ce-NT in Figure $3 \mathrm{~g}$, the single hyperfine in Figure $\mathrm{S} 8$ indicates only the $\mathrm{Ce}^{3+}-\mathrm{O}-\mathrm{Ce}^{4+}$ defect $^{32}$ at a negligible concentration. For the 2D-3D CeO $2-x$ in Figure $3 g$, the hyperfines in Figure S9 reveal the presence of $\mathrm{Ce}^{3+}, \mathrm{O}_{2}^{-}$, and $\mathrm{Ce}^{3+}-\mathrm{O}^{-}-\mathrm{Ce}^{4+}$ defects at higher concentrations. These relative concentrations were derived from the total peak areas (using the baselines) of the second integrals of the EPR signal. For the Mn$\mathrm{Ce}$ and $\mathrm{Cu}-\mathrm{Ce}$ in Figure $3(\mathrm{~h}, \mathrm{i})$, the respective hyperfines in Figures S10 and S11 reveal that fabrication of the heterojunction nanostructures introduces new defects associated with TMOs at even higher concentrations than were detected for 2D-3D $\mathrm{CeO}_{2-\mathrm{x} \text {. }}$

The hyperfine interaction between electrons and nuclei provides information concerning the number and identity of ions and their distances from unpaired electrons. $\mathrm{Mn}$ and $\mathrm{Cu}$, with electron configurations of $3 d^{2}$ and $3 d^{3}$, exhibit four 
and six absorption signals, respectively, as shown in Figures S10 and S11. Regarding Ce (with electron configurations of $4 f^{1}$ and $\left.5 p^{6}\right), \mathrm{Ce}^{4+}$ has no unpaired electrons (EPR-inactive), while $\mathrm{Ce}^{3+}$ has a single unpaired electron (EPR-active). However, other EPR peaks can be generated by other unpaired electrons resulting from $V_{O}^{*}, V_{O}^{*}, V_{O}^{x}, \mathrm{O}_{2}^{-}$, and $\mathrm{Ce}^{3+}-\mathrm{O}^{-}-\mathrm{Ce}^{4+}{ }^{29}$. Table $\mathrm{S} 2$ provides the $g$ factors corresponding to the observed hyperfines. The 2D-3D $\mathrm{CeO}_{2-x}, \mathrm{Cu}-\mathrm{Ce}$, and $\mathrm{Mn}-\mathrm{Ce}$ exhibit six hyperfine that correspond to values characteristic of the defect $\mathrm{Ce}^{3+}-\mathrm{O}^{-32}$, albeit with g-factors shifted to lower values relative to that of 2.00 for the free electron of Ce-NT. For the $\mathrm{Ce}-\mathrm{Mn}$ and $\mathrm{Ce}-\mathrm{Cu}$, shifted g-factors are effectively the maximal that can be attributed to the $\mathrm{Mn}\left(3 d^{2}\right)$ and $\mathrm{Cu}\left(3 d^{3}\right)$ species, the EPR signals of which are manifested in the form of shoulders on the sharp peaks and rounded humps with the hyperfines. Figures S8-S11 assess the first and second integrals of the hyperfines for all of the nanostructures, revealing the defect and charge carrier concentrations, which are assumed to be electrons as $\mathrm{CeO}_{2-x}$ is an $n$-type semiconductor, although it may exhibit $p$-type semiconductivity under some peculiar conditions, owing to the known presence of cerium vacancies $\left(V_{C e}^{\prime \prime \prime \prime}\right) .^{9}$

Further, The decrease in the first integral is owing to the effect of the energy levels of the trapped electrons, while such decrease in the second integral reflects the balance of the annihilation and creation of defects resulting from the introduction of the TMOs. Figure $3 \mathrm{c}$ shows that the $\left[\mathrm{V}_{O}^{*}\right]$ for the TMO-decorated heterostructures were greater than that for the 2D-3D mesoporous $\mathrm{CeO}_{2-x}$, which suggests that other defects were annihilated. These were likely surface structural defects that were blocked by the apposition of the TMO particles. This result is supported by the decrease in surface areas of the decorated heterostructures, as shown in Figure 3f. Another source of defects is intervalence charge transfer (IVCT), which can both create and eliminate defect states. ${ }^{33}$

CO-Conversion Catalysis and DFT Simulation. Figure 4a provides the catalytic performance by the nanostructures in terms of the temperatures for 10 , 50 , and $90 \%$ CO conversion $\left(T_{10 \%}, T_{50 \%}, T_{90 \%}\right)$. The $T_{10 \%}$ value for the Ce-NT sample was $160^{\circ} \mathrm{C}$ and the $\mathrm{T}_{90 \%}$ was $372^{\circ} \mathrm{C}$, which are consistent with previous reports for pristine micron-sized $\mathrm{CeO}_{2} \cdot{ }^{34,} 35$ In contrast, the 2D-3D $\mathrm{CeO}_{2-x}$ exhibited an outstanding performance, with a $\mathrm{T}_{90 \%}$ of $148^{\circ} \mathrm{C}$, which is the lowest temperature among the best-performing pristine $\mathrm{CeO}_{2-x}$ nanostructures, as shown by the red line in Figure $4 \mathrm{~b}$. The data for $\mathrm{Mn}-\mathrm{Ce}$ and $\mathrm{Cu}-\mathrm{Ce}$ in Figure $4 \mathrm{a}$ show that these heterostructures exhibit $\mathrm{T}_{90 \%}$ values of $124^{\circ} \mathrm{C}$ and $88^{\circ} \mathrm{C}$, respectively delivering even better catalytic performance relative to the $2 \mathrm{D}-3 \mathrm{D} \mathrm{CeO}_{2-x}$ nanostructure. The blue line in Figure $4 \mathrm{~b}$ shows that $\mathrm{Cu}-\mathrm{Ce}$ in this work offers a lower $\mathrm{T}_{90 \%}$ values than that demonstrated by a selection of the best metal- or oxide- $\mathrm{CeO}_{2-x}$ heterostructures available. Comparative lists of the best $\mathrm{CeO}_{2}$ and $\mathrm{CeO}_{2}$-based nanostructures, their characteristics, and cited references are given in Tables S3 and $\mathrm{S4}$, respectively. 

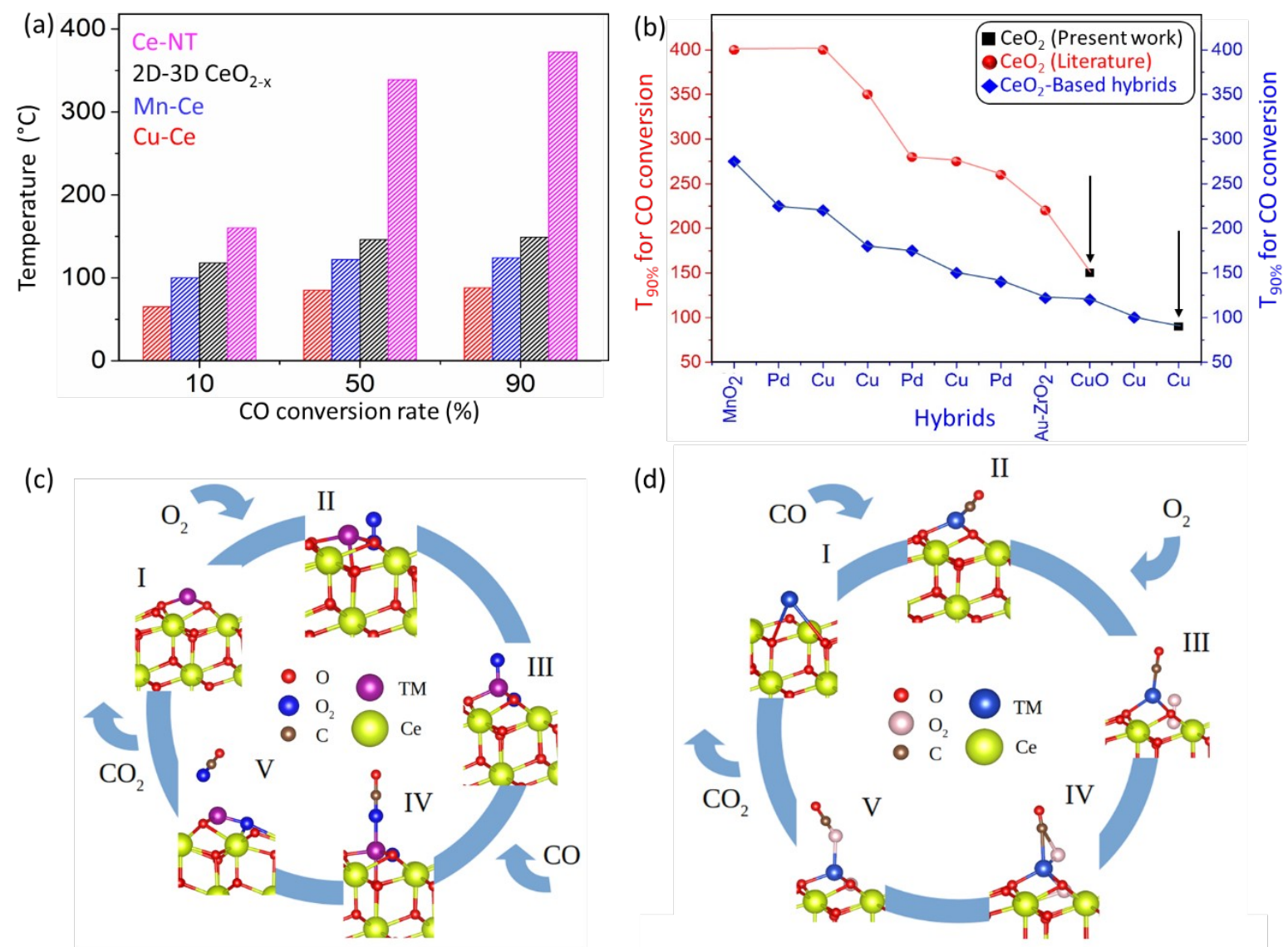

(d)
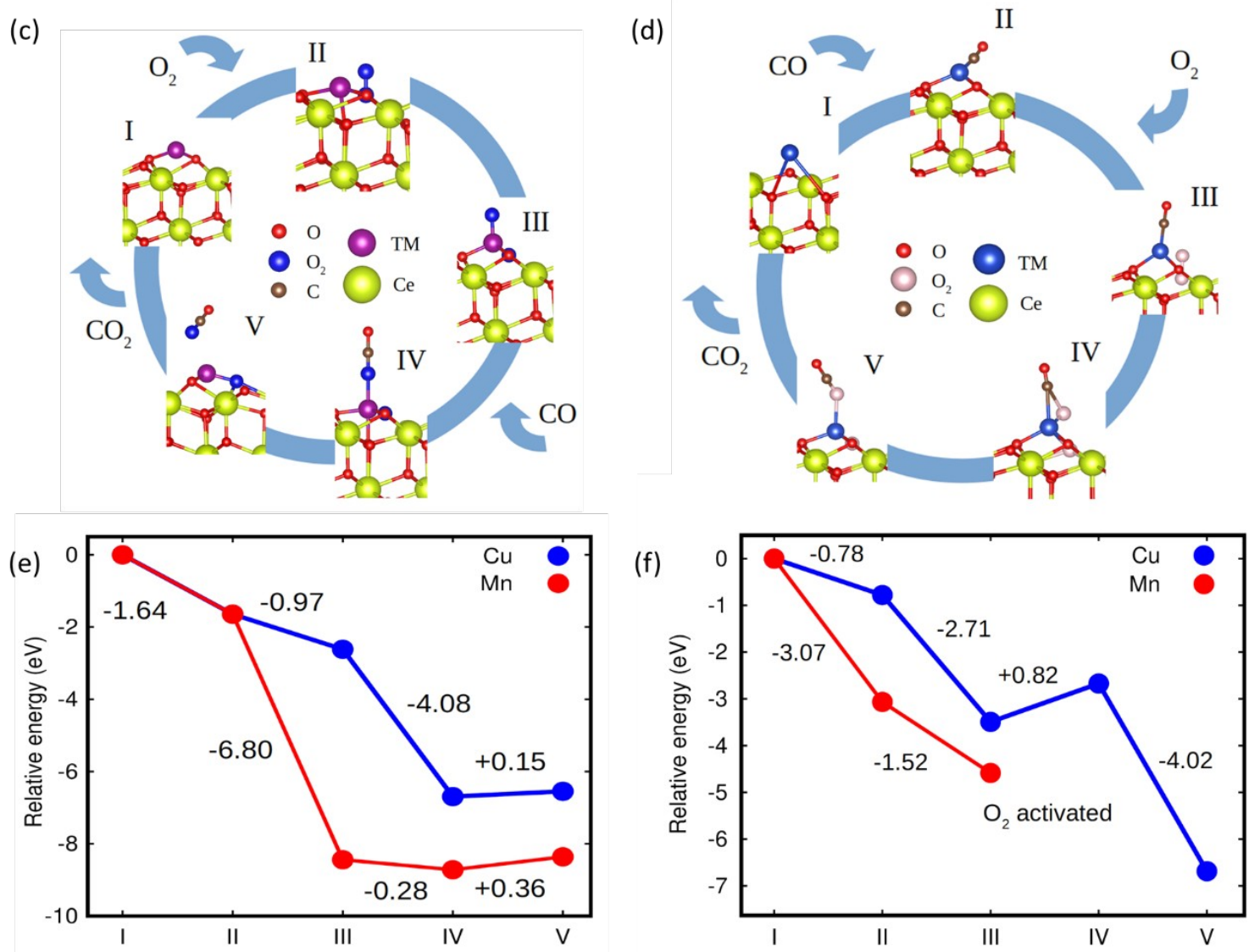

Figure 4. (a) $\mathrm{CO}$ oxidation plots for $\mathrm{Ce}-\mathrm{NT}, \mathrm{CeO}_{2-x}, \mathrm{Mn}-\mathrm{Ce}, \mathrm{Cu}-\mathrm{Ce}$. (b) Comparative $\mathrm{CO}$ oxidation data for $\mathrm{CeO}_{2-x}$ and $\mathrm{TMO}-$ decorated $\mathrm{CeO}_{2-x}$. (c) Mechanism 1: CO-oxidation reaction path with initial $\mathrm{O}_{2}$ adsorption. (d) Mechanism 2: CO-oxidation reaction path with initial CO adsorption. (e) Energy profiles calculated for Mechanism 1. (f) Energy profiles calculated for Mechanism 2.

Although the Cu-Ce nanostructure has a lower specific surface area (SSA) relative to those of the $2 \mathrm{D}-3 \mathrm{D} \mathrm{CeO}_{2-x}$ and $\mathrm{Mn}$-Ce nanostructures (Table S1), the former exhibits the highest $\mathrm{CO}$ conversion rate at the lowest temperature. This apparent contradiction has been examined by first-principles density functional theory (DFT) calculations of $\mathrm{CO}$ and $\mathrm{O}_{2}$ molecule adsorption on $\mathrm{MnO}$ - and CuOdecorated $\mathrm{CeO}_{2-x}$. The calculations reveal that the energetically most favorable sites for $\mathrm{O}_{2}$ and $\mathrm{CO}$ adsorption are in the $V_{O}^{\cdot *}$ sites on the $\mathrm{CeO}_{2-x}$ surface and on 
the TM ions, respectively. Consequently, depending on the order of adsorption of the molecules, there are two different possible $\mathrm{CO}$ oxidation mechanisms: initial $\mathrm{O}_{2}$ adsorption in the $V_{O}^{\cdot *}$ (Figure $4 \mathrm{c}$ ) or initial $\mathrm{CO}$ adsorption on the TM ions on the $\mathrm{CeO}_{2-x}$ surface (Figure $4 d$ ).

Mechanism 1: Initially (Step I), an $\mathrm{O}_{2}$ molecule from the atmosphere is immobilized in an oxygen vacancy adjacent to a TM ion on the $\mathrm{CeO}_{2-x}$ surface (Step II). Since spontaneous dissociation of the $\mathrm{O}_{2}$ molecule (which would preclude quenching by $\mathrm{O}_{2}$ adsorption) and subsequent adsorption of one of the oxygen ions on the TM ion is energetically most favorable, this exothermic process (Figure 4e) takes place (Step III). Owing to the strong attraction between this adsorbed oxygen ion and a $\mathrm{CO}$ molecule, the introduction of $\mathrm{CO}$ gas results in its adsorption on this oxygen ion (Step IV). This adsorption is favored with a Cu ion relative to an $\mathrm{Mn}$ ion (Figure 4e). Finally, the comparative desorption energies of the $\mathrm{CO}_{2}$ molecule from the $\mathrm{Mn}$ ion $(0.36 \mathrm{eV})$ and from the $\mathrm{Cu}$ ion $(0.15 \mathrm{eV})$ demonstrates that desorption from the $\mathrm{Mn}$ ion is less favored than from the $\mathrm{Cu}$ ion. As these are endothermic processes, they require energy inputs, hence the required $\mathrm{T}_{90 \%}$ values of $124^{\circ} \mathrm{C}$ and $88^{\circ} \mathrm{C}$, respectively.

Mechanism 2: Initially (Step I), a CO molecule is immobilized a TM ion on the $\mathrm{CeO}_{2-x}$ surface (Step II). Subsequently, an $\mathrm{O}_{2}$ molecule from the atmosphere is immobilized in an oxygen vacancy adjacent to a TM ion (Step III). In this case, the dissociation of the $\mathrm{O}_{2}$ molecule and subsequent adsorption of one of the oxygen ions to form a $\mathrm{CO}_{2}$ molecule on a TM ion is energetically unfavorable (Figure 4f). In the case of the $\mathrm{Cu}$ ion, oxygen ion adsorption is endothermic $(0.82$ eV) and so occurs upon heating (Step IV). In contrast, with the $\mathrm{Mn}$ ion, the more significant difference in $\mathrm{Mn}-\mathrm{C}$ electronegativity than for $\mathrm{Cu}-\mathrm{C}$ suggests stronger attraction between the former pair of ions, which suppresses the potential for oxygen ion adsorption to the carbon ion to form the $\mathrm{CO}_{2}$ molecule. Consequently, with sufficient heating, the $\mathrm{O}_{2}$ molecule dissociates but adsorption does not occur. In the case of the $\mathrm{Cu}$ ion, once the $\mathrm{CO}_{2}$ molecule is formed, $\mathrm{CO}_{2}$ desorption (Step V) occurs readily owing to its highly exothermic nature (Figure 4f).

The comparative analysis of the DFT results indicates that Mechanism 1 is likely to be the dominant process in the CO oxidation. Consequently, the low $\mathrm{T}_{90 \%}$ value for $\mathrm{Cu}$-Ce relative to those reported elsewhere (Table S4) reflects the energetically favorable reaction sequence of three exothermic adsorption reactions followed by a very low endothermic desorption reaction, even though $\mathrm{Cu}-\mathrm{Ce}$ has the lower SSA of the two heterostructures (Table S1).

\section{SUMMARY}

The present work introduces a simplified and template-free technique to fabricate multidimensional mesoporous nanostructures. The approach utilizes a Ce-based coordination polymer precursor of stratified structure that can be exfoliated rapidly. During subsequent heating, the stacked-layer precursor converts into macro-mesoporous 2D-3D $\mathrm{CeO}_{2-x}$, depending on control of the kinetics of the transformation. The resultant nanostructures show ultra-high surface areas, defect densities, and pore volumes, which are responsible for unparalleled catalytic performance for $\mathrm{CO}$ conversion. The performance was improved further by modifying the 2D-3D $\mathrm{CeO}_{2-x}$ via $\mathrm{TMO}$ decoration to form heterojunction nanostructures. The enhanced catalytic activities upon using the 
TMO modifier, were investigated systematically using structural analyses and first principles DFT simulations. This technique offers new directions for costeffective and highly controllable fabrication of advanced multidimensional nanocatalysts. 


\section{- ASSOCIATED CONTENT Supporting information}

The Supporting Information is available free of charge on the ACS publications website.

Included are experimental section, figures describing zeta potential, SEM and EDX images of the heterojunction nanostructures, XRD of 2D-3D $\mathrm{CeO}_{2-x}, \mathrm{Ce}-\mathrm{NT}$, $\mathrm{Mn}-\mathrm{Ce}$, and $\mathrm{Cu}-\mathrm{Ce}$, data related to EPR analysis and peak assignments for 2D-3D $\mathrm{CeO}_{2-x}, \mathrm{Ce}-\mathrm{NT}, \mathrm{Mn}-\mathrm{Ce}$, and $\mathrm{Cu}-\mathrm{Ce}$; a table providing CO-conversion results obtained here and compared with other selected best-reported catalysts.

\section{- AUTHOR INFORMATION \\ Corresponding Authors}

Sajjad S. Mofarah -- School of Materials Science and Engineering, UNSW Sydney, Sydney, NSW 2052, Australia;Orcid.org/0000-0002-4835-767X;

Email: s.seifimofarah@unsw.edu.au

Charles C. Sorrell -- School of Materials Science and Engineering, UNSW Sydney, Sydney, NSW 2052, Australia;

c.sorrell@unsw.edu.au

\section{Authors}

Luisa Schreck -- School of Materials Science and Engineering, UNSW Sydney, Sydney, NSW 2052, Australia

Claudio Cazorla -- School of Materials Science and Engineering, UNSW Sydney, Sydney, NSW 2052, Australia

Xiaoran Zheng -- School of Materials Science and Engineering, UNSW Sydney, Sydney, NSW 2052, Australia

Esmaeil Adabifiroozjaei -- Research Center for Functional Materials (RCFM), National Institute for Materials Science (NIMS), Tsukuba, Ibaraki 305-0047, Japan

Constantine Tsounis -- Particles and Catalysis Research Group, School of Chemical Engineering, UNSW Sydney, Sydney, NSW 2052, Australia.

Jason Scott -- Particles and Catalysis Research Group, School of Chemical Engineering, UNSW Sydney, Sydney, NSW 2052, Australia.

Reza Shahmiri -- School of Materials Science and Engineering, UNSW Sydney, Sydney, NSW 2052, Australia

Yin Yao -- Electron Microscopy Unit (EMU), Mark Wainwright Analytical Centre, UNSW Sydney, Sydney, NSW 2052, Australia

Roozbeh Abbasi -- School of Chemical Engineering, UNSW Sydney, NSW 2052, Australia

Kourosh Kalantar-Zadeh -- School of Chemical Engineering, UNSW Sydney, NSW 2052, Australia

Yuan Wang -- School of Chemistry, Faculty of Science, The University of New South Wales, Sydney, NSW 2052, Australia

Hamidreza Arandiyan -- Laboratory of Advanced Catalysis for Sustainability, School of Chemistry, University of Sydney, NSW 2006, Australia

Leigh Sheppard -- School of Engineering, Western Sydney University, Penrith, NSW, 2751, Australia; orcid.org/0000-0003-3549-6969

Esmail Doustkhah -- International Center for Materials Nanoarchitechtonics (MANA), National Institute for Materials Science (NIMS), 1-1 Namiki, Tsukuba, Ibaraki 305-0044, Japan 
Pramod Koshy -- School of Materials Science and Engineering, UNSW Sydney, Sydney, NSW 2052, Australia

\section{Author Contributions}

S.S.M designed the project, conducted most of the experiments, characterization, provided the first draft, and worked on all the corresponding revisions. L.S. synthesized heterojunction $\mathrm{Mn}-\mathrm{Ce}$ and $\mathrm{Cu}-\mathrm{Ce}$ nanostructures and assisted with SEM imaging. C.C. implemented the first-principles simulations, wrote the related section, and commented on the final version of the draft. E.A. performed EELS characterization and commented on the final version of the draft. C.T. and J.S. conducted the CO-conversion tests and provided catalytic results. R.S. conducted the EPR tests and provided the corresponding analysis. Y.Y. conducted AFM imaging. R.A. assisted with experimental design and revision of the drafts. K.K.Z. commented on all the drafts and revised the final versions. Y.W. and H.A. assisted with analyzing the catalytic performance, commented, and worked on the final versions of the draft. L.S. assisted in setting up the electrochemical cell, conducting Ce-CP synthesis, and revision of the drafts. E.D. performed SSA test for Ce-NT and commented on the final version of the draft. P.K. contributed to the data analyses and revised final version of the manuscript. C.C.S. worked on all drafts of the manuscript and supervised the overall project.

\section{Funding}

This work has been supported by the Australian Research Council (DP170104130). The authors are grateful for access to the characterization facilities provided by the Mark Wainwright Analytical Centre, UNSW Sydney and beam time at the Wombat beamline of the Australian Nuclear Science and Technology Organization (ANSTO). S.S.M. is pleased to acknowledge UIPA and RTP scholarship support from UNSW Sydney. E.A. Acknowledges the financial support (JSPS KAKENHI Grant Number: 18F18064) provided by the Japan Society for the Promotion of Science.

\section{Notes}

The authors declare no competing financial interest.

\section{ACKNOWLEDGMENTS}

We acknowledge the Mark Wainwright Analytical Centre, Sydney, Australia for provision of characterization facilities and assisting with data analysis. Further, we acknowledge Setareh M. Pour for providing the schematic images in this work. 


\section{- REFERENCES}

(1) Zhang, Q.; Lee I.; Ge J.; Zaera F.; Yin Y. Surface-Protected Etching of Mesoporous Oxide Shells for the Stablization of Metal Nanocatalysts. Adv. Func. Mater. 2010, 20 (14), 2201-2214.

(2) Doustkhah E.; Ide Y.; Bursting Exfoliation of a Microporous Layered Silicate to Three-Dimenssionally Meso-Microporous Nanosheets for Improved Molecular Recognition. ACS Appl. Nano. Mater. 2019, 2 (12), 7513-7520.

(3) Shen, K.; Zhang, L.; Chen, X.; Liu, L.; Zhang, D.; Han, Y.; Chen, J.; Long, J.; Luque, R.; Li, Y.; Chen, B. Ordered Macro-Microporous Metal-Organic Framework Single Crystals. Science 2018, 359 (6372), 206-210.

(4) Lan, K.; Liu, Y.; Zhang, W.; Liu, Y.; Elzatahry, A.; Wang, R.; Xia, Y.; AlDhayan, D.; Zheng, N.; Zhao, D. Uniform Ordered Two-Dimensional Mesoporous $\mathrm{TiO}_{2}$ Nanosheets from Hydrothermal-Induced Solvent-Confined Monomicelle Assembly. J. Am. Chem. Soc. 2018, 140 (11), 4135-4143.

(5) Peng, L.; Fang, Z.; Li, J.; Wang, L.; Bruck, A. M.; Zhu, Y.; Zhang, Y.; Takeuchi, K. J.; Marschilok, A. C.; Stach, E. A.; Takeuchi, E. S.; Yu, G. TwoDimensional Holey Nanoarchitectures Created by Confined Self-Assembly of Nanoparticles via Block Copolymers: From Synthesis to Energy Storage Property. ACS nano 2018, 12 (1), 820-828.

(6) Xiong, J.; Di, J.; Xia, J. X.; Zhu, W. S.; Li, H. M. Surface Defect Engineering in 2D Nanomaterials for Photocatalysis. Adv. Funct. Mater. 2018, 28, 39.

(7) Voiry, D.; Shin, H. S.; Loh, K. P.; Chhowalla, M. Low-Dimensional Catalysts for Hydrogen Evolution and $\mathrm{CO}_{2}$ Reduction. Nat. Rev. Chem. 2018, 2, 0105.

(8) Voiry, D.; Shin, H. S.; Loh, K. P.; Chhowalla, M. Low-Dimensional Catalysts for Hydrogen Evolution and $\mathrm{CO}_{2}$ Reduction. Nat. Rev. Chem. 2018, 2, 0105.

(9) Mofarah, S. S.; Adabifiroozjaei, E.; Pardehkhorram, R.; Assadi, M. H. N.; Hinterstein, M.; Yao, Y.; Liu, X.; Ghasemian, M. B.; Kalantar-Zadeh, K.; Mehmood, R.; Cazorla, C.; Shahmiri, R.; Bahmanrokh, G.; Bhattacharyya, S.; Spadaro, M. C.; Arbiol, J.; Lim, S.; Xu, Y.; Arandiyan, H.; Scott, J.; Koshy, P.; Sorrell, C. C. Coordination Polymer to Atomically Thin, Holey, Metal-Oxide Nanosheets for Tuning Band Alignment. Adv. Mater. 2019, 31 (52), 1905288.

(10) Peng, L.; Fang, Z.; Zhu, Y.; Yan, C.; Yu, G. Holey 2D Nanomaterials for Electrochemical Energy Storage. Nat. Energy 2018, 8 (9), 1702179.

(11) Peng, L.; Xiong, P.; Ma, L.; Yuan, Y.; Zhu, Y.; Chen, D.; Luo, X.; Lu, J.; Amine, K.; Yu, G. Holey Two-Dimensional Transition Metal Oxide Nanosheets For Efficient Energy Storage. Nat. Commun. 2017, 8, 15139.

(12) Wen, W.; Wu, J. M.; Cao, M. H. Facile Synthesis of a Mesoporous $\mathrm{Co}_{3} \mathrm{O}_{4}$ Network for Li-Storage via Thermal Decomposition of an Amorphous Metal Complex. Nanoscale 2014, 6, (21), 12476-12481.

(13) Zhang, G.; Ren, L.; Yan, Z.; Kang, L.; Lei, Z.; Xu, H.; Shi, F.; Liu, Z. H. Rational Design and Controllable Preparation of Holey $\mathrm{MnO}_{2}$ Nanosheets. Chem. Commun. 2017, 53 (20), 2950-2953.

(14) Bo, X.; Li, Y.; Hocking, R. K.; Zhao, C. NiFeCr Hydroxide Holey Nanosheet as Advanced Electrocatalyst for Water Oxidation. ACS Appl. Mater. Inter. 2017, 9 (47), 41239-41245.

(15) Fang, Z.; Peng, L.; Qian, Y.; Zhang, X.; Xie, Y.; Cha, J. J.; Yu, G. Dual Tuning of Ni-Co-A ( $A=P$, Se, O) Nanosheets by Anion Substitution and Holey Engineering for Efficient Hydrogen Evolution. J. Am. Chem. Soc. 2018, 140 (15), 5241-5247.

(16) Sun, Y.; Liu, Q.; Gao, S.; Cheng, H.; Lei, F.; Sun, Z.; Jiang, Y.; Su, H.; Wei, S.; Xie, Y. Pits Confined in Ultrathin Cerium(IV) Oxide for Studying Catalytic Centers in Carbon Monoxide Oxidation. Nat. Commun. 2013, 4, 2899.

(17) Yu, T.; Lim, B.; Xia, Y. Aqueous-Phase Synthesis of Single-Crystal Ceria Nanosheets. Angew. Chem. Int. Ed. 2010, 49 (26), 4484-4487. 
(18) S. Mofarah, S.; Adabifiroozjaei, E.; Wang, Y.; Arandiyan, H.; Pardehkhorram, R.; Yao, Y.; Assadi, M. H. N.; Mehmood, R.; Chen, W.-F.; Tsounis, C.; Scott, J.; Lim, S.; Webster, R.; Zhong, V.; Xu, Y.; Koshy, P.; Sorrell, C. C. Assembly of Cerium-Based Coordination Polymer into Variant Polycrystalline 2D3D $\mathrm{CeO}_{2-x}$ Nanostructures. J. Mater. Chem. A 2020, 8, 4753-4763.

(19) Zhu, Z.; Yin, H.; He, C.T.; Al-Mamun, M.; Liu, P.; Jiang, L.; Zhao, Y.; Wang, Y.; Yang, H.G.; Tang, Z.; Wang, D.; Chen, X. M.; Zhao H. Ultrathin Transition Metal Dichalcogenide/3d Metal Hydroxide Hybridized Nanosheets to Enhance Hydrogen Evolution Activity. Adv. Mater. 2018, 30(28), 1801171.

(20) Zou, F.; Hu, X.; Li, Z.; Qie, L.; Hu, C.; Zeng, R.; Jiang, Y.; Huang, Y. MOFDerived Porous $\mathrm{ZnO} / \mathrm{ZnFe}_{2} \mathrm{O}_{4} / \mathrm{C}$ Octahedra with Hollow Interiors for High-Rate Lithium-Ion Batteries. Adv. Mater. 2014, 26 (38), 6622-8.

(21) Salunkhe, R. R.; Tang, J.; Kamachi, Y.; Nakato, T.; Kim, J. H.; Yamauchi, Y. Asymmetric Supercapacitors Using 3D Nanoporous Carbon and Cobalt Oxide Electrodes Synthesized from a Single Metal-Organic Framework. ACS Nano 2015, 9 (6), 6288-6296.

(22) Zhang, F.; Chen, C.; Xiao, W.-m.; Xu, L.; Zhang, N. CuO/CeO ${ }_{2}$ Catalysts with Well-Dispersed Active Sites Prepared from $\mathrm{Cu}_{3}(\mathrm{BTC})_{2}$ Metal-Organic Framework Precursor for Preferential CO Oxidation. Catal. Commun. 2012, 26, 25-29.

(23) Mehmood, R.; Wang, X.; Koshy, P.; Yang, J. L.; Sorrell, C. C. Engineering Oxygen Vacancies through Construction of Morphology Maps for Bio-Responsive Nanoceria for Osteosarcoma Therapy. CrystEngComm 2018, 20 (11), 1536-1545.

(24) Feng, B.; Sugiyama, I.; Hojo, H.; Ohta, H.; Shibata, N.; Ikuhara, Y. Atomic Structures and Oxygen Dynamics of $\mathrm{CeO}_{2}$ Grain Boundaries. Sci. Rep. 2016, 6, 20288.

(25) Mofarah, S. S.; Adabifiroozjaei, E.; Yao, Y.; Koshy, P.; Lim, S.; Webster, R.; Liu, X.; Khayyam Nekouei, R.; Cazorla, C.; Liu, Z.; Wang, Y.; Lambropoulos, N.; Sorrell, C. C. Proton-Assisted Creation of Controllable Volumetric Oxygen Vacancies in Ultrathin $\mathrm{CeO}_{2-x}$ for Pseudocapacitive Energy Storage Applications. Nat. Commun. 2019, 10, 2594.

(26) Wu, Z.; Li, M.; Howe, J.; Meyer, H. M.; Overbury, S. H. Probing Defect Sites on $\mathrm{CeO}_{2}$ Nanocrystals with Well-Defined Surface Planes by Raman Spectroscopy and $\mathrm{O}_{2}$ Adsorption. Langmuir 2010, 26 (21), 16595-16606.

(27) Kosacki, I.; Suzuki, T.; Anderson, H. U.; Colomban, P. Raman Scattering and Lattice Defects in Nanocrystalline $\mathrm{CeO}_{2}$ Thin Films. Solid State Ion. 2002, 149, 99-105.

(28) Spanier, J. E.; Robinson, R. D.; Zhang, F.; Chan, S.-W.; Herman, I. P. SizeDependent Properties ofCeO $2-y$ Nanoparticles as Studied by Raman Scattering. Phys. Rev. B 2001, 64 (24), 2454071-8.

(29) López, J. M.; Gilbank, A. L.; García, T.; Solsona, B.; Agouram, S.; TorrenteMurciano, L. The Prevalence of Surface Oxygen Vacancies Over the Mobility of Bulk Oxygen in Nanostructured Ceria for the Total Toluene Oxidation. Appl. Catal. B: Environ. 2015, 174-175, 403-412.

(30) Ilton, E. S.; Post, J. E.; Heaney, P. J.; Ling, F. T.; Kerisit, S. N. XPS Determination of Mn Oxidation States in Mn (hydr)oxides. Appl. Surf. Sci. 2016, 366, 475-485.

(31) Zhang, Q.; Huang, L.; Kang, S.; Yin, C.; Ma, Z.; Cui, L.; Wang, Y. CuO/Cu ${ }_{2} \mathrm{O}$ Nanowire Arrays Grafted by Reduced Graphene Oxide: Synthesis, Characterization, and Application in Photocatalytic Reduction of $\mathrm{CO}_{2}$. RSC Adv. 2017, 7 (69), 43642-43647.

(32) Wang, L.; Yu, Y.; He, H.; Zhang, Y.; Qin, X.; Wang, B. Oxygen Vacancy Clusters Essential for the Catalytic Activity of $\mathrm{CeO}_{2}$ Nanocubes for o-xylene Oxidation. Sci Rep. 2017, 7, 12845. 
(33) Chen, W. F.; Mofarah, S. S.; Hanaor, D. A. H.; Koshy, P.; Chen, H. K.; Jiang, Y.; Sorrell, C. C. Enhancement of $\mathrm{Ce} / \mathrm{Cr}$ Codopant Solubility and Chemical Homogeneity in $\mathrm{TiO}_{2}$ Nanoparticles through Sol-Gel versus Pechini Syntheses. Inorg. Chem. 2018, 57 (12), 7279-7289.

(34) Zhou, X.; Ling, J.; Sun, W.; Shen, Z. Fabrication of Homogeneously $\mathrm{Cu}^{2+} / \mathrm{La}^{3+}$-Doped $\mathrm{CeO}_{2}$ Nanosheets and their Application in $\mathrm{CO}$ Oxidation. J. Mater. Chem. A 2017, 5 (20), 9717-9722.

(35) Kim, K.; Yoo, J. D.; Lee, S.; Bae, M.; Bae, J.; Jung, W.; Han, J. W. Simple Descriptor to Rapidly Screen CO Oxidation Activity on Rare-Earth Metal-Doped $\mathrm{CeO}_{2}$ : From Experiment to First-Principles. ACS Appl. Mater. Inter. 2017, 9 (18), 15449-15458. 


\section{Graphic Abstract}

The present work reports a new template-free, high-yield, and flexible approach to fabricate centimeter-scale, 3D, mesoporous, $\mathrm{CeO}_{2-x}$ based nanostructures that are comprised of holey 2D nanosheets as thin as $\sim 4 \mathrm{~nm}$. The method involves conversion of a newly developed, bulk-layered, Ce-based coordination polymer by controlled kinetics of removal of organic species. Further, the flexibility of the synthesis approach allows fabrication of various TMO-based heterojunction nanostructures with outstanding surface areas and densities of active sites, which yield superior $\mathrm{CO}$ conversion performance.

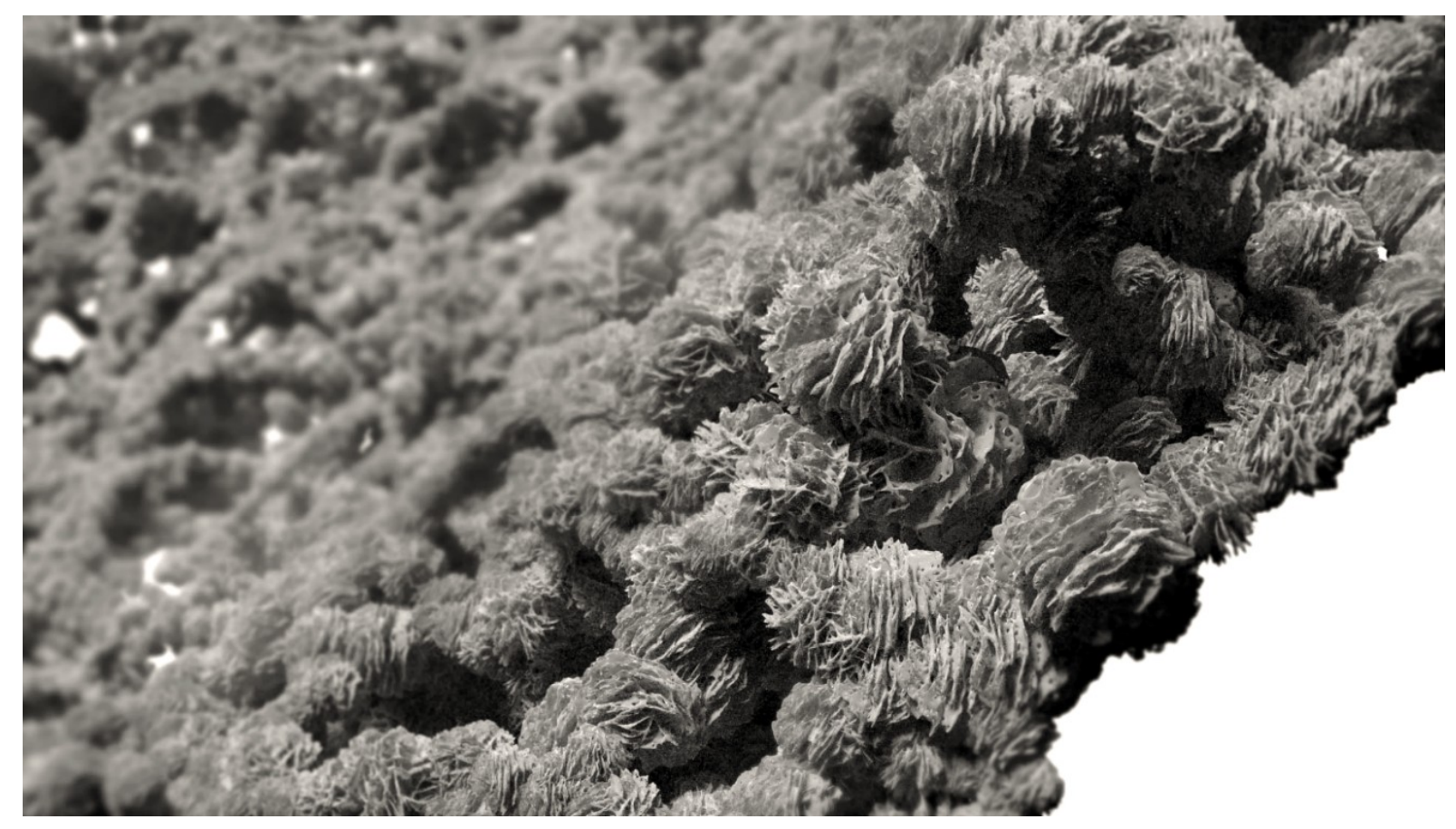

